\title{
A Control Approach to High-Speed Probe-Based Nanofabrication
}

\author{
Yan Yan, Qingze Zou ${ }^{\dagger}$ and Zhiqun Lin
}

\begin{abstract}
In this paper, an inversion-based feedforward control approach to achieve high-speed, large-range probe-based nanofabrication is proposed. Probe-based nanofabrication has attracted great interests recently. However, this technique is still limited by the low-throughput due to the challenges in compensating for the adverse effects such as the nonlinear hysteresis and the vibrational dynamics of piezo actuators in each axis, as well as the dynamics coupling in multi-axis motion during high-speed nanofabrication. The main contribution of this article is the utilization of the recently-developed model-less inversion-based iterative control (MIIC) technique to overcome these challenges in SPM probe-based nanofabrication. By using this advanced control technique, precision position control of the probe can be achieved during high-speed, large-range multiaxes nanofabrication. The proposed approach is demonstrated in experiments by implementing it to fabricate large-size $(\sim 50 \mu \mathrm{m})$ pentagram patterns via mechanical-scratching on a gold-coated silicon sample surface at high speed $(\sim 4.5 \mathrm{~mm} / \mathrm{sec})$.
\end{abstract}

\section{INTRODUCTION}

In this paper, an iterative feedforward control approach to achieve high-speed, large-range, probe-based nanofabrication is presented. Recently, probe-based nanofabrication using tools such as scanning probe microscope (SPM) has attracted many interests. However, current probe-based nanofabrication processes [1]-[3] are limited by the low-throughput of the process, which, in turn, hurdles their practical implementations. Although such a low-throughput can be improved through hardware improvements such as parallel probes [3], the throughput is eventually limited if the fabrication speed is low due to the hardware adverse effects, which can lead to large positioning errors of the probe relative to the sample during high-speed, large-range fabrication, and then, large defects in the nano-pattern/parts fabricated. The contribution of this article is the implementation of a recently-developed inversion-based iterative control approach [4] to compensate for the adverse hardware effects during high-speed, largerange probe-based nanofabrication. The proposed approach is illustrated by nanofabricating patterns on a gold-coated silicon sample surface via mechanical scratching. Experimental results are presented and discussed to demonstrate that the proposed method can effectively compensate for the hardware adverse effects, thereby substantially improve the speed (throughput) in probe-based nanofabrication.

Precision positioning during high-speed, large-range motion is needed in probe-based nanofabrication. It has become evident that probe-based methods are a promising approach

The authors are with the Department of Mechanical Engineering (Yan Yan and Qingze Zou) and the Department of Material Science and Engineering (Zhiqun Lin), Iowa state University, Ames, IA, 50011 USA. †Corresponding author, E-mail: qzzou@iastate.edu. to fabricating nanoscale structures and devices (because of their low cost and significant technical potential) [5]. Various nanofabrication processes have been proposed [1]-[3], [6]. All of these processes require the precision positioning of the probe relative to the sample, thereby, being confronted by the same challenge-maintaining precision (probe-tosample) positioning during high-speed large-range operation. This is because large positioning errors can be generated during high-speed, large-range fabrication, which will not only lead to large defects in the fabricated structures or devices, but also result in damage of the probe (when the sample is hard), the sample (when the sample is soft), or both. Moreover, the motion control can be very demanding in all 3-D axes for probe-based nanofabrication. In addition, the dynamics coupling of the piezo actuators cross multi-axis can be large when the fabrication speed is high and/or the operation range is large, which results in large fabrication distortions as well. Therefore, it is important to maintain the precision positioning of the probe relative to the sample in all $x-y-z$ axes during high-speed, large-size nanofabrication.

Advanced control techniques can be used to improve the positioning precision during high-speed, large-range motion. For example, it has been demonstrated recently that the output tracking in repetitive operations can be substantially improved by using the inversion-based iterative control (IIC) techniques. A main advantage of iterative control approach is the exploitation of the noncausality gained from the repetitive nature of the applications, particularly for nonminimumphase systems such as piezo actuators in SPM. Also, it has been shown recently that the IIC approach can compensate for both hysteresis and dynamics effects of piezo actuators. The model-less iterative control (MIIC) employed in this article further extends the IIC approach, by eliminating the need for the dynamics model in the control algorithm. Therefore, constraints related to the modeling process as well as the need for a good dynamics model are removed. We note that in nanofabrication, the desired trajectory is usually specified a priori, and the environment tends to be well maintained. Therefore, it is advantageous to utilize iterative control techniques such as MIIC in probe-based nanofabrication.

The main contribution of the article is the use of the MIIC technique to the probe-based nanofabrication using SPM. Particularly, the MIIC technique is utilized to compensate for the dynamics-coupling effect in multi-axis motions, as well as to account for the hysteresis and the dynamics effects in the motion of each individual $x, y$ and $z$ axes. The approach is illustrated by implementing it to fabricate a challenging pattern (pentagram) via mechanical-scratching on a gold- 
coated silicon sample surface. Furthermore, a dashed-line pentagram pattern was also fabricated, and the experimental results obtained demonstrate the efficacy of the proposed method for high-speed 3-D nanofabrication.

\section{MIIC APPROACH TO PROBE-BASED NANOFRABRICATION}

In this section, the MIIC technique [4] is presented. We start with briefly describing the probe-based nanofabrication.

\section{A. Probe-Based Nanofabrication}

In probe-based nanofabrication, a micro-machined probe is precisely positioned relative to the sample surface while the probe is moved on or closely-above the sample surface (see Fig. 1) to locally induce surface modification along the path, resulting in nanoscale features on the sample surface (for example, lines or dots). Such a surface modification can be achieved, for example, through mechanical scratching followed by an etching process [1], or, through thermal effect as exemplified in the IBM Millipede system [3]. Alternatively, probe-based nanofabrication can also be achieved by introducing external effects such as electrical field or laser beam [6], as well as chemical compound (via probe coating) [2]. In all these nanofabrication mechanisms, it is crucial to maintain the precision of the probe-to-sample relative position, because the error of probe-to-sample position directly translates to the defects in the fabricated nanostructures/devices. Such a precision positioning becomes challenging when the nanofabrication is conducted at high speed and large range, because of the excitation of the adverse dynamics effects - the cross-axis dynamics coupling, the hysteresis, as well as the vibrational dynamics effects.

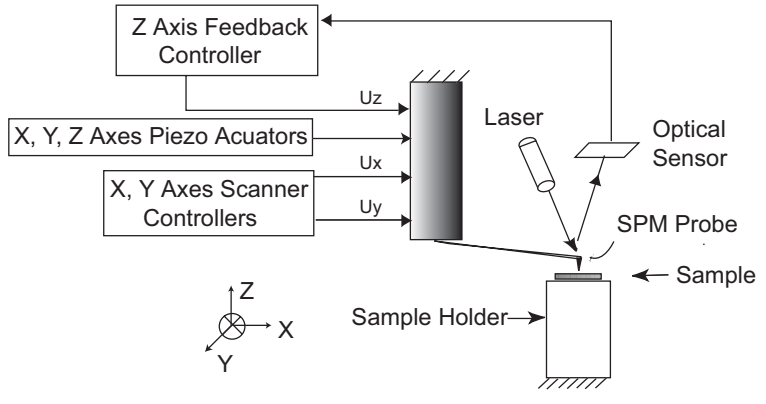

Fig. 1. The schematic diagram of SPM system.

\section{B. MIIC Approach to Multi-axis Motion Control}

We propose to utilize the recently-developed MIIC approach to compensate for the above adverse effects during the probe-based nanofabrication. Iterative learning control (ILC) approach is attractive because in nanofabrication, usually the desired trajectories for all $x-y-z$ axes are prespecified and repetitive. Therefore, it is possible to utilize the tracking result of the entire trajectory from the previous iteration to generate the control input at current time instant, rather than relying on the tracking result before current time instant only - as in feedback control. In other words, ILC approach provides the possibility to explore the noncausality in nanofabrication to enhance the precision positioning.
The MIIC algorithm is described in frequency domain as

$$
\begin{aligned}
u_{0}(j \omega)= & \alpha y_{d}(j \omega) \\
u_{k+1}(j \omega)= & \frac{u_{k}(j \omega)}{y_{k}(j \omega)} y_{d}(j \omega), \\
& \left(\text { for } u_{k}(j \omega) \neq 0, y_{k}(j \omega) \neq 0, k \geq 1\right)
\end{aligned}
$$

where $\alpha \neq 0$ is a pre-chosen constant (e.g., $\alpha$ can be chosen as the estimated DC-gain). $f(j \omega)$ denotes the Fourier transform of the signal $f(t) \cdot y_{d}(j \omega)$ is the desired output trajectory, and $u_{k}(j \omega)$ and $y_{k}(j \omega)$ are the input and the output obtained from the $k^{\text {th }}$ iteration, respectively.

\section{Compensating for the Cross-Axis Coupling Effect}

Compensating for the cross-axis dynamics coupling existing in multi-axis motion control [7]. is particularly important when fabricating 3-D nano-devices/patterns, because the motions in all $x-y-z$ axes can be complicated and at high speed. Although such cross-axis coupling effects can be accounted for by considering the SPM dynamics in $x-y-z$ axes as a multi-input-multi-output (MIMO) system and designing a MIMO controller accordingly [8], such an approach, requires complicated online computations, and its performance is limited by the possibly large model uncertainties. In this article, MIIC algorithm is used to compensate for not only the dynamics and hysteresis effects of the piezo actuator in each axis (for the $z$-axis, the dynamics effect also comes from the cantilever along with the mechanical fixture connecting the piezo actuator to the cantilever), but also the cross-axis dynamics coupling effects. Such a cross-axis coupling effect is pronounced from the large-range lateral $x-y$ axes motion to the vertical $z$-axis motion, and more significant in highspeed operation. As schematically shown in Fig. 2, the $x$ $y$-to- $z$ coupling-caused displacement $y_{z x y}(j \omega)$ is augmented to the displacement for $z$-axis tracking $y_{z z}(j \omega)$ in the total $z$-axis displacement $y_{z}(j \omega)$,

$$
\begin{aligned}
\begin{aligned}
y_{z}(j \omega) \stackrel{G_{z z}}{ }(j \omega) u_{z}(j \omega)+G_{z x}(j \omega) u_{x}(j \omega) \\
+
\end{aligned} & G_{z y} u_{y}(j \omega) \\
\triangleq & y_{z z}(j \omega)+y_{z x y}(j \omega) . \\
& \left(y_{z x y}(j \omega) \triangleq G_{z x}(j \omega) u_{x}(j \omega)+G_{z y}(j \omega) u_{y}(j \omega)\right)
\end{aligned}
$$

Fig. 2. The $z$-axis SPM dynamics with $x$-y-to- $z$ coupling effect.

Thus first, the $x-y$-to- $z$ coupling-caused displacement $y_{z x y}(j \omega)$ is measured by applying the control input to the lateral $x-y$ axes on a hard flat reference sample (e.g., a silicon sample or a sapphire calibration sample). Then, the desired $z$-axis displacement $y_{z, d}(j \omega)$ is modified by subtracting with the measured coupling-caused displacement $y_{z x y}(j \omega)$,

$$
\hat{y}_{z, d}(j \omega)=y_{z, d}(j \omega)-y_{z x y}(j \omega),
$$


and the MIIC technique is applied to the $z$-axis only (with no lateral displacement, i.e., $y_{z x y}(j \omega)=0$ in (3)) to find the control input $u_{z}^{*}(j \omega)$ that tracks the modified $z$-axis desired trajectory, i.e.,

$$
G_{z z}(j \omega) u_{z}^{*}(j \omega)=y_{z z}(j \omega) \longrightarrow \hat{y}_{z, d}(j \omega)
$$

Finally, the control inputs for both the lateral $x-y$ and $z$-axis tracking are applied simultaneously. As a result, the $x-y$-to- $z$ coupling is removed and precision positioning in all $x-y-z$ axes is achieved.

\section{EXPERIMENTAL EXAMPLE}

We illustrate the MIIC approach to probe-based nanofabrication through experiments. We start with briefly describing the nanofabrication process based on mechanical scratching.

\section{A. Nanofabrication Based on Mechanical Scratching}

In this paper, we use mechanical scratching to directly create patterns on a gold coated silicon sample. The experiments were carried out under ambient condition on a SPM system (Dimension 3100, Veeco Inc.) with a rectangularshape cantilever coated with wear-resistant material. The nominal stiffness of the probe is $40 \mathrm{~N} / \mathrm{m}$ (stiffer probes like the stainless steel cantilever with diamond tip can be used to further reduce weariness and increase the smoothness of the fabricated pattern). By applying a relatively large loading force to the SPM probe on the sample surface, and dragging the probe to track the desired geometry path, patterns of nanoscale feature can be fabricated. Then afterwards, the fabricated pattern can be examined by imaging the sample surface using the same SPM system with a substantially lower loading force. We note that as discussed before, the precision positioning problem ubiquitously exists in probebased nanofabrication processes, thereby, we expect that the proposed MIIC technique can be equally applied to other probe-based nanofabrication processes as well.

\section{B. Experimental setup}

The SPM system utilized in this article uses piezotube actuators to position the SPM probe with respect to the sample in all $x-y-z$ axes. All the control inputs were generated by using MATLAB-xPC-target (Mathworks, Inc.), and sent through a data acquisition card to the high-voltage amplifier of the SPM-controller directly.

In the following experiments, two types of pentagram (one with continuous lines, and the other with dashed lines) were chosen as the desired patterns to be fabricated. When fabricating the continuous-line pentagram pattern, contact mode SPM was used to maintain the cantilever deflection around a set point value (i.e., to maintain a constant tipsample interaction force), by using the feedback controller of the SPM system for the $z$-axis probe positioning. When fabricating the dashed-line pentagram pattern, the $z$-axis feedback control was turned off, and the vertical position of the $z$-axis piezo actuator was controlled by applying the feedforward input obtained from the MIIC technique to track the desired $z$-axis trajectory. The fabrication of the dashed-line pentagram pattern required the up-and-down vertical motion of the probe. Thus, such an experiment evaluated the MIIC algorithm for fabricating 3-D structures. The desired pentagram pattern (size: $50 \mu \mathrm{m}$ by $50 \mu \mathrm{m}$ ) and the corresponding desired trajectories for each $z, x$, and $y$ axis are shown in Fig. 3 (a), (b), (c), and (d), respectively. Particularly, an isosceles trapezoidal wave was chosen as the desired $z$-axis waveform. The use of the isosceles trapezoidal wave rather than square wave was to reduce the oscillations after the up-down transitions. The entire pentagram pattern composed a total of 20 dash lines evenly spaced.
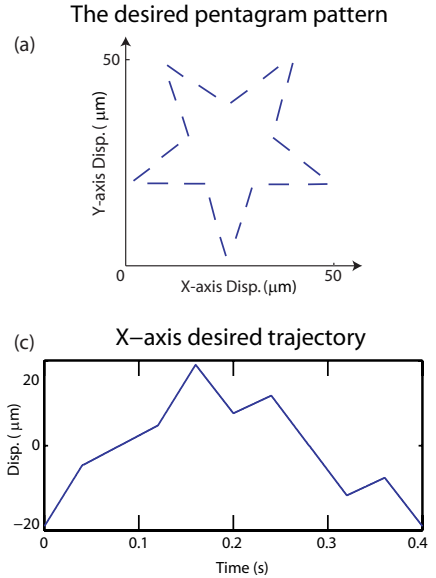
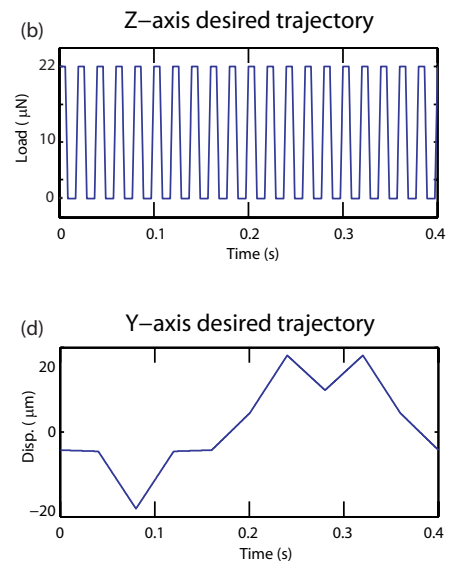

Fig. 3. The desired trajectories of the dash-line pentagram pattern: the entire trajectory (a), the $z$-axis trajectory (b), the $x$-axis trajectory (c), and the $y$-axis trajectory $(\mathrm{d})$.

\section{Tracking Results and Discussion}

In the experiments, the MIIC technique was applied to achieve precision tracking in all $x-y-z$ axes simultaneously, and then, the converged inputs were used to fabricate the pattern by applying a large load force $(\sim 22 \mu \mathrm{N})$ to the cantilever. For comparison, we also used the DC-gain method to fabricate the pentagram pattern, where the control input was generated by scaling the desired output with the DC-gain of the piezo actuator. The DC-gain method does not account for the hysteresis nor the vibrational dynamics effects, thereby the patterns fabricated demonstrate these adverse effects on the fabrication quality.

To compensate for the $x-y$-to- $z$ coupling effect, the coupling-caused $z$-axis displacement was measured from the cantilever deflection when pressing the probe onto a hard silicon sample of nanoscale flatnessand applying the $x$-axis and $y$-axis control inputs for tracking the respective $x, y$ axis desired trajectories (see Fig.3 (c), (d))). Then the $z$ axis desired trajectory (see Fig.3 (b)) was subtracted by this measured coupling-caused displacement as the new desired trajectory, and the MIIC algorithm was used to obtain the control input to track this modified $z$-axis desired trajectory. Finally, this control input was applied to z-axis when the control inputs to the other two axes were applied simultaneously. We note that for this SPM system, the dynamicscoupling effect from vertical $z$-axis to lateral $x-y$ axes and between $x$ and $y$ axes were small and negligible.

The experimental tracking results in all $x-y-z$ axes were acquired and compared. Three different fabrication rates (5 $\mathrm{Hz}, 15 \mathrm{~Hz}$ and $25 \mathrm{~Hz}$ ) were tested in the experiment, where 
the fabrication rate was defined to be the rate to finish the fabrication of the entire pattern once. The corresponding average lateral speed for the three fabrication rates were at $\sim 0.9 \mathrm{~mm} / \mathrm{sec}, \sim 2.7 \mathrm{~mm} / \mathrm{sec}$, and $\sim 4.5 \mathrm{~mm} / \mathrm{sec}$, respectively. At these three fabrication rates, the corresponding $z$-axis waveform frequency (for fabricating the dashed-line pentagram pattern) was 100,300 , and $500 \mathrm{~Hz}$, respectively. In Fig. 4, the lateral $x$-axis tracking results for the fabrication rates of $5 \mathrm{~Hz}$ and $25 \mathrm{~Hz}$ obtained by using the converged MIIC inputs are compared with the desired trajectory as well as those obtained by using the DC-gain method. To evaluate the $z$-axis tracking (with no lateral $x-y$ axes motion), the modified desired trajectory was used (to account for the $x-y$ to- $z$ coupling) when the MIIC was applied, and the original desired trajectory was used when the DC-gain method was used. During the implementation of the MIIC technique, the iterations were stopped when all the $x-y-z$ axes sensor signals converged to the desired tracking trajectories. The $z-$ axis tracking results obtained by using the converged MIIC input are compared with the modified desired trajectory in Fig. 5 for the waveform frequencies of $100 \mathrm{~Hz}$ and $500 \mathrm{~Hz}$ (corresponding to the fabrication rates of $5 \mathrm{~Hz}$ and $25 \mathrm{~Hz}$, respectively). The $z$-axis tracking results obtained by using the DC-gain method are compared with the original desired trajectory in Fig. 6. Finally, the $z$-axis tracking during the fabrication process (i.e., when all $x-y-z$ axes inputs were applied simultaneously) was also compared for the MIIC method, the DC-gain method against the original desired trajectory, as shown in Fig. 7.

The tracking performance was also evaluated by quantifying the relative RMS error and the relative maximum error of the tracking results, as listed in Table. I for the lateral tracking and Table. II for the vertical tracking, where the relative maximum tracking error $E_{M}(\%)$, and the relative RMS error $E_{R M S}(\%)$ are defined as

$$
\begin{gathered}
E_{M}(\%) \triangleq \frac{\left\|y_{d}(\cdot)-y_{k}(\cdot)\right\|_{\infty}}{\left\|y_{d}(\cdot)\right\|_{\infty}} \times 100 \% \\
E_{R M S}(\%) \triangleq \frac{\left\|y_{d}(\cdot)-y_{k}(\cdot)\right\|_{2}}{\left\|y_{d}(\cdot)\right\|_{2}} \times 100 \% .
\end{gathered}
$$

Discussion The experimental results demonstrate that precision positioning in lateral $x-y$ axes motion can be achieved by using the MIIC algorithm during large-range, high-speed nanofabrication. As the lateral displacement range was large $(50 \mu \mathrm{m})$ in the experiments, the hysteresis effect was pronounced, and large positioning errors were generated. As shown in Fig. 4 (a1) and (a2), with the DC-gain method, the hysteresis-caused relative maximum error $E_{M}(\%)$ was over $10 \%$ of the total displacement range when the fabrication rate was slow $(5 \mathrm{~Hz})$. Such a large positioning error was substantially reduced by using the MIIC algorithm. As the fabrication rate was increased to $25 \mathrm{~Hz}$, the vibrational dynamics effect was augmented to the hysteresis effect, resulting in even larger tracking errors. However, precision tracking was still maintained when using the MIIC approach. We note that for nanofabrication application, precision tracking in $x$ and $y$ axes are equally crucial, because even if the tracking error in each individual axis is small, a relatively large distortion can still be generated in the final fabricated pattern. Such an "amplification" of positioning error is caused by the superposition of the errors in different axes. In the experiment, precision tracking in $y$ axis was also achieved by using the MIIC technique. The simultaneous precision tracking in both $x$ and $y$ axes leaded to the precision fabrication of the continuous pentagram pattern.
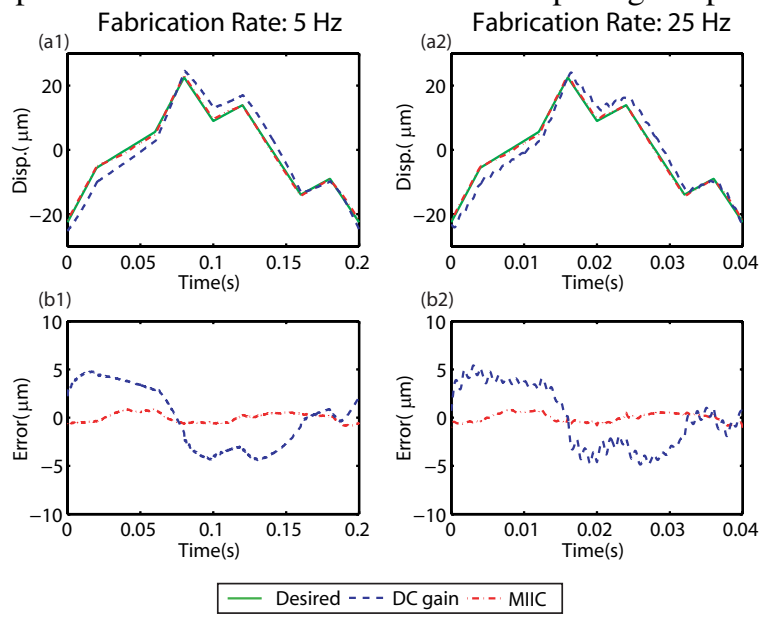

Fig. 4. Comparison of the lateral $x$ tracking results obtained by using the MIIC technique with that by using the DC-gain method at (a1) $5 \mathrm{~Hz}$, (a2) $25 \mathrm{~Hz}$, and comparison of the corresponding tracking errors at (b1) $5 \mathrm{~Hz}$, (b2) $25 \mathrm{~Hz}$, respectively.
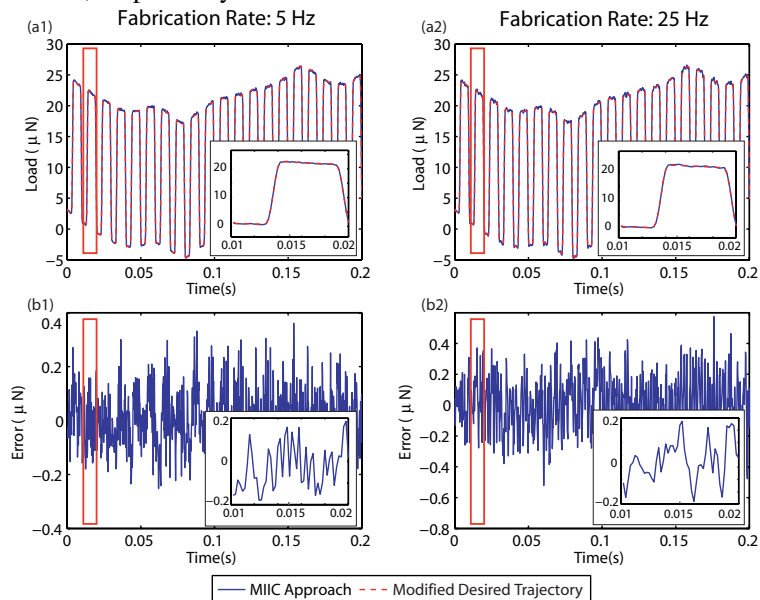

Fig. 5. Comparison of the vertical $z$ tracking results by using the MIIC technique with the modified desired trajectory at (a1) $5 \mathrm{~Hz}$, (a2) $25 \mathrm{~Hz}$, and comparison of the corresponding tracking errors at (b1) $5 \mathrm{~Hz}$ (b1), (b2) 25 $\mathrm{Hz}$, respectively.

When fabricating the dashed-line pentagram pattern, the frequency of the isosceles trapezoidal wave was much higher (20 times higher) than that in the lateral $x-y$ axes. As a result, large probe oscillations in the vertical $z$-axis not only increased the roughness of the sample surface, but can also further damaged the probe, the sample or both. By using the MIIC algorithm, however, even at the fabrication rate of $25 \mathrm{~Hz}$, precision tracking was still achieved in the vertical $z$-axis tracking. Thus, the MIIC algorithm can effectively account for adverse effects during high-speed, large-size nanofabrication in both lateral and vertical directions.

The experimental results also showed that dynamicscoupling-caused disturbance in multi-axis motion can also be 

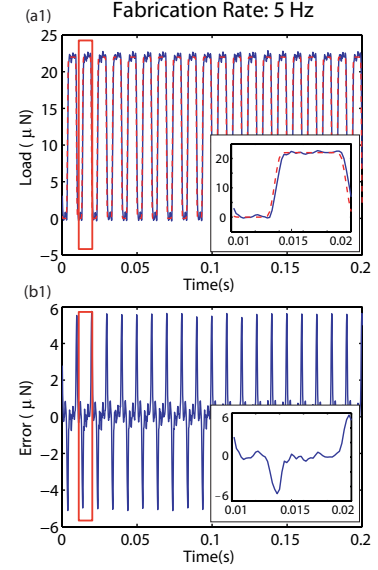

- DC-gain Approach - - - Original Desired Trajectory

Fig. 6. Comparison of the vertical $z$ tracking results by using the DC-gain method with the original desired trajectory at (a1) $5 \mathrm{~Hz}$, (a2) $25 \mathrm{~Hz}$, and comparison of the corresponding tracking errors at (b1) $5 \mathrm{~Hz}$, (b2) $25 \mathrm{~Hz}$, respectively.

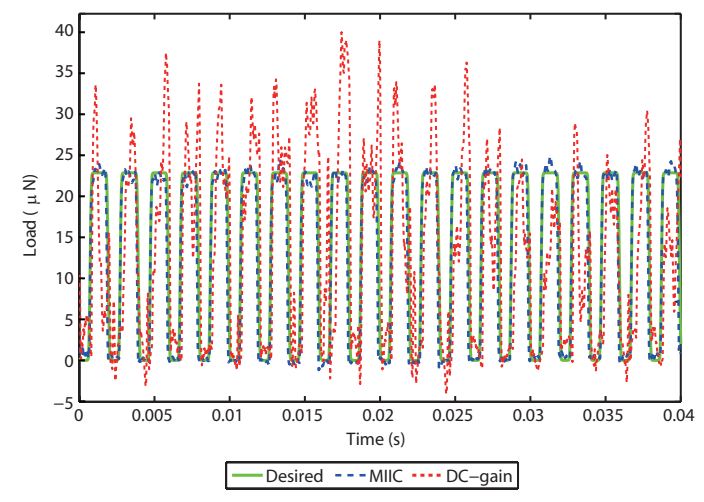

Fig. 7. Comparison of the $z$-axis deflection signal by using the MIIC technique with that by using the DC-gain method at $25 \mathrm{~Hz}$ during the fabrication process (i.e., when lateral $x-y$ axes inputs were also applied simultaneously).

TABLE I

COMPARISON OF THE TRACKING ERRORS IN $X$-AXIS OBTAINED BY USING THE DC-GAIN METHOD, AND THE MIIC APPROACH AT DIFFERENT FABRICATION RATES.

\begin{tabular}{c|ccc|ccc}
\hline \hline Error & \multicolumn{3}{|c|}{$E_{M}(\%)$} & \multicolumn{3}{c}{$E_{R M S}(\%)$} \\
\hline Fab. Rate & $\mathbf{5 ~ H z}$ & $\mathbf{1 5} \mathbf{~ H z}$ & $\mathbf{2 5} \mathbf{~ H z}$ & $\mathbf{5} \mathbf{~ H z}$ & $\mathbf{1 5} \mathbf{~ H z}$ & $\mathbf{2 5} \mathbf{~ H z}$ \\
\hline Speed $(\mathbf{m m} / \mathbf{s})$ & $\mathbf{0 . 6 4}$ & $\mathbf{1 . 9 3}$ & $\mathbf{3 . 2 1}$ & $\mathbf{0 . 6 4}$ & $\mathbf{1 . 9 3}$ & $\mathbf{3 . 2 1}$ \\
\hline $1^{\text {st }}$ Iter. & 3.81 & 3.67 & 4.23 & 1.49 & 1.39 & 1.41 \\
$2^{\text {nd }}$ Iter. & 2.04 & 2.12 & 2.11 & 1.15 & 1.12 & 1.20 \\
$3^{\text {rd }}$ Iter. & 1.80 & 1.90 & 2.44 & 1.09 & 1.05 & 1.13 \\
$4^{\text {th }}$ Iter. & 1.88 & 1.97 & 2.30 & 0.99 & 1.09 & 1.14 \\
\hline DC-gain & 10.66 & 11.53 & 12.05 & 7.02 & 6.92 & 7.16 \\
\hline \hline
\end{tabular}

TABLE II

COMPARISON OF THE TRACKING ERRORS IN Z-AXIS OBTAINED BY USING THE DC-GAIN METHOD, AND THE MIIC APPROACH AT DIFFERENT FABRICATION RATES.

\begin{tabular}{c|ccc|ccc}
\hline \hline Error & \multicolumn{3}{|c|}{$E_{M}(\%)$} & \multicolumn{3}{c}{$E_{R M S}(\%)$} \\
\hline Fab. Rate & $\mathbf{5 ~ H z}$ & $\mathbf{1 5} \mathbf{~ H z}$ & $\mathbf{2 5 ~} \mathbf{~ H z}$ & $\mathbf{5 ~} \mathbf{~ H z}$ & $\mathbf{1 5} \mathbf{~ H z}$ & $\mathbf{2 5 ~} \mathbf{~ H z}$ \\
\hline $1^{\text {st }}$ Iter. & 5.18 & 5.84 & 7.57 & 0.78 & 0.86 & 1.80 \\
$2^{\text {nd }}$ Iter. & 2.03 & 2.30 & 3.28 & 0.55 & 0.64 & 0.87 \\
$3^{\text {rd }}$ Iter. & 1.14 & 1.20 & 2.06 & 0.34 & 0.44 & 0.50 \\
$4^{\text {th }}$ Iter. & 1.16 & 1.18 & 2.21 & 0.34 & 0.43 & 0.57 \\
\hline DC-gain & 26.15 & 52.81 & 74.82 & 9.07 & 18.48 & 28.31 \\
\hline \hline
\end{tabular}
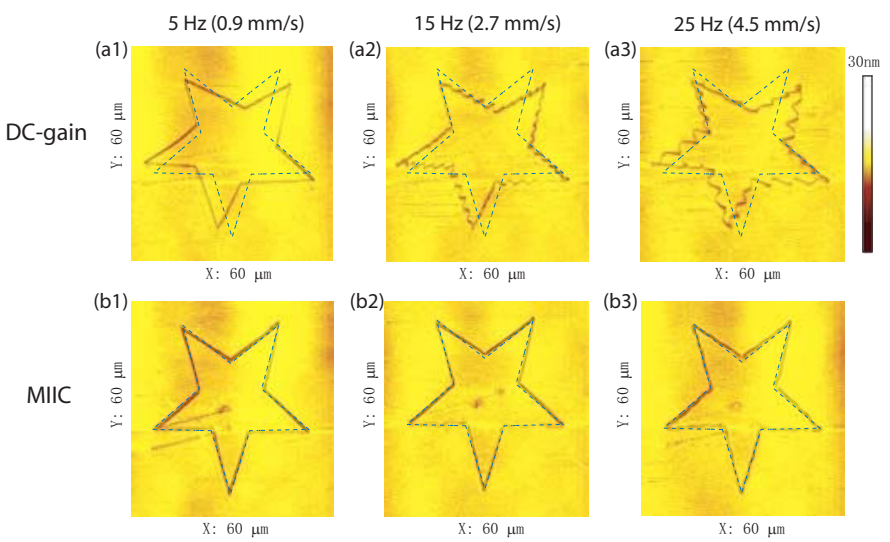

Fig. 8. Comparison of the nanofabrication images of the continuous pentagram pattern obtained by using (top row) the DC-gain method with (bottom row) those obtained by using the MIIC technique at (a1, b1) $5 \mathrm{~Hz}$, (a2, b2) $15 \mathrm{~Hz}$, and (a3, b3) $25 \mathrm{~Hz}$, respectively. The blue-dashed lines represent the desired pentagram pattern.
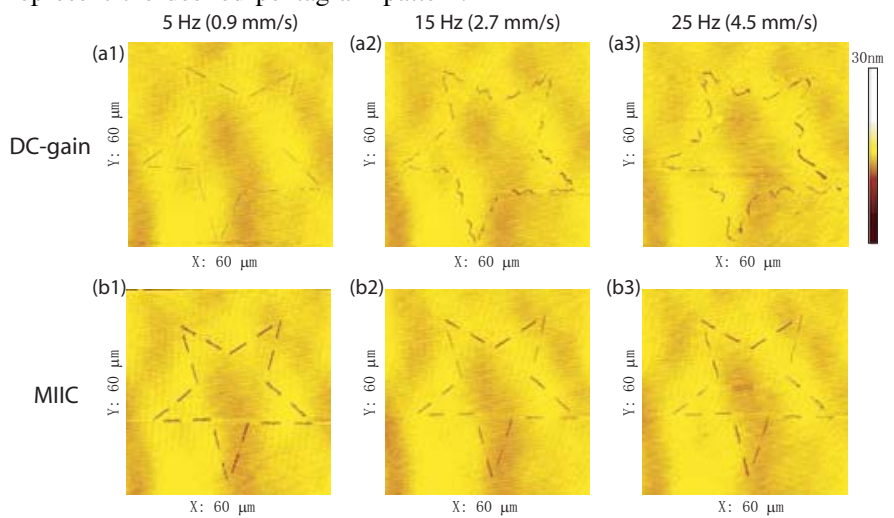

Fig. 9. Comparison of the nanofabrication images of the dashed-line pentagram pattern obtained by (top row) using the DC-gain method with (bottom row) those obtained by using the MIIC technique at (a1, b1) $5 \mathrm{~Hz}$, (a2, b2) $15 \mathrm{~Hz}$, and (a3, b3) $25 \mathrm{~Hz}$, respectively.

effectively removed by using the proposed method. Comparing the modified $z$-axis desired trajectory (in Fig. 5) with the original one (in Fig. 6), we can see that the $x$-y-to- $z$ coupling effect is pronounced. The coupling-caused $z$-axis displacement was around $40 \%$ of the (original) desired trajectory when the lateral $x-y$ axes motion was large-range $(50 \mu \mathrm{m})$ and high-speed $(4.5 \mathrm{~mm} / \mathrm{sec})$. Such large coupling-caused disturbance was augmented to the vibrational dynamics effect when all 3-D inputs were applied during the nanofabrication of the dashed-line pentagram, resulting in much larger tracking error (than that if there were no coupling effect). On the contrary, such a large coupling-caused disturbance was removed with the use of the proposed MIIC technique, and precision tracking of the original $z$-axis desired trajectory was achieved during the 3-D nanofabrication. Therefore, the experimental results demonstrate that the proposed approach can achieve high-speed precision positioning in 3-D probebased nanofabrication at large-size.

\section{Nanofabrication Results and Discussion}

Nanofabrication of continuous-line pentagram pattern Next, to fabricate the continuous-line pentagram pattern, the MIIC inputs were applied to the $x$ and $y$ axes simultaneously with a larger load force. Then the fabricated sample area was 
imaged immediately afterwards on the same SPM system. The SPM images of the fabricated patterns obtained by using the MIIC technique are compared with those obtained by using the DC-gain method in Fig. 8 for the three fabrication rates $(5 \mathrm{~Hz}, 15 \mathrm{~Hz}$ and $25 \mathrm{~Hz})$. For comparison, the desired pentagram pattern was also marked by the blue-dashed line in Fig. 8. As shown in Fig. 8, the MIIC technique effectively removed the fabrication distortion caused by the hysteresis and the vibrational dynamics effects. When the fabrication speed was relatively slow at $5 \mathrm{~Hz}$ (the corresponding averaged line speed was $\sim 0.9 \mathrm{~mm} / \mathrm{sec}$, see Fig. 8 (a1)), the distortion in the fabricated pattern was already obvious. Such pronounced distortions were mainly caused by the nonlinear hysteresis effect existing in both $x$ - and $y$ - axes piezo actuators (because the fabrication size was large $(50 \mu \mathrm{m})$. Particularly, the positioning errors were amplified due to the superimposition of the $x$-axis errors with the $y$-axis ones, caused mainly by the phase-delay and asynchronization between the $x$ and the $y$ axes. In contrast, the use of the MIIC approach effectively removed such fabrication distortions (see Fig. 8 (b1)). When the fabrication rate was increased to $15 \mathrm{~Hz}$ (the corresponding averaged line speed was $\sim 2.7 \mathrm{~mm} / \mathrm{sec}$ ), the tracking error caused by the vibrational dynamics became significant, resulting in large oscillatory distortions in the fabricated pattern when the DC-gain method was used (see Fig. 8 (a2)), which became even larger when the fabrication rate was further increased to $25 \mathrm{~Hz}$ (with an averaged line speed at $4.5 \mathrm{~mm} / \mathrm{sec}$, see Fig. 8 (a3)). Using the MIIC technique, precision tracking was still maintained during this high-speed, large-range fabrication. As shown in Fig. 8 (b2), (b3), the pattern distortion was very small, and the fabricated pattern almost overlapped with the desired one (marked by the blue-dashed line in Fig. 8). Therefore, the experimental results demonstrate that by using the MIIC approach, highspeed probe-based nanofabrication of 2-D pattern can be achieved.

Nanofabrication of the dashed-line pentagram pattern Next, the converged MIIC inputs for all the $x-y-z$ axes were applied to the $x, y$, and $z$ axes respectively at the same time (the $z$-axis feedback control was turned off), and the dashedline pentagram pattern was fabricated. Then the fabricated sample area was imaged immediately afterwards on the same SPM. The SPM images of the fabricated patterns obtained by using the MIIC technique are compared with those obtained by using the DC-gain method (applied to all 3-D axes) in Fig. 9 for the three fabrication rates $(5 \mathrm{~Hz}, 15 \mathrm{~Hz}$ and 25 $\mathrm{Hz}$ ). To avoid confusion, the desired pentagram pattern was not marked out in Fig. 9. We also examined the indentation depth of the dashed-line. As shown by the cross-section plot in Fig. 10, the indentation depth was $\sim 10 \mathrm{~nm}$. The experimental results showed the efficacy of the proposed approach in achieving 3-D precision positioning during highspeed probe-based nanofabrication. When the fabrication rate was at $5 \mathrm{~Hz}$, the distortions caused by nonlinear hysteresis and vibrational dynamics effects were already pronounced. As shown in Fig. 9 (a1), the dashed-lines in the pattern obtained by using the DC-gain method were curved (rather than straight) and varied in length. However, such large fabrication errors in the dashed lines were significantly reduced by using the MIIC method. When the rate was increased to $15 \mathrm{~Hz}$ and $25 \mathrm{~Hz}$, the hysteresis and dynamics caused pattern distortions became much more severe. As shown in Fig. 9 (a2, a3), the dashed lines were more curved and largely varied in length. Such large pattern distortions were substantially reduced by using the proposed method. Therefore, the experimental results demonstrated that the MIIC approach can be effectively utilized for high-speed nanofabrication of large-size 3-D patterns.

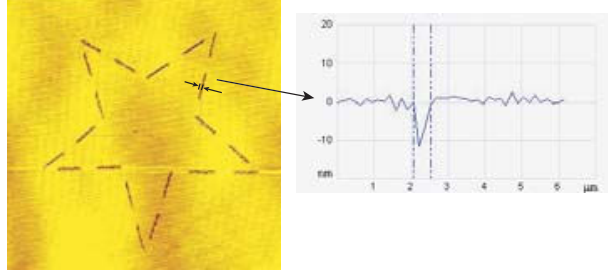

Fig. 10. The cross section image of the dashed pentagram. The left image shows the depth of the fabricated groove.

\section{CONCLUSION}

A control approach to achieve probe-based high-speed nanofabrication at large range has been proposed. The implementation of the MIIC technique to the SPM probe-based nanofabrication can effectively compensate for the nonlinear hysteresis and vibrational dynamics effects of piezotube actuator, thereby improve the fabrication throughput. The approach has been illustrated by implementing it to fabricate a pentagram via mechanical-scratching on a gold-coated silicon sample surface. The experimental results showed that the pentagram patterns of a size of $\sim 50 \mu \mathrm{m}$ with both continuous and dashed-lines can be accurately fabricated at a high (averaged) line speed of $4.5 \mathrm{~mm} / \mathrm{sec}$.

\section{ACKNOWLEDGMENTS}

Zou would like to acknowledge the financial supports from NSF Grants CMMI 0626417 and DUE 0632908.

\section{REFERENCES}

[1] R. Magno and B. R. Bennett, "Nanostructure patterns written in iii$v$ semiconductors by an atomic force microscope," Appl. Phys. Lett., vol. 70, p. 1855, April, 1997.

[2] R. D. Piner, J. Zhu, F. Xu, S. Hong, and C. A. Mirkin, "Dip-Pen nanolithography," Science, vol. 283, no. 5402, pp. 661-663, 1999

[3] Vettiger, G. Cross, M. Despont, U. Drechsler, U. Durig, B. Gotsmann, W. Haberle, M. A. Lantz, H. E. Rothuizen, R. Stutz, and G. Binnig, "The millipede - nanotechnology entering data storage," IEEE, vol. 1, pp. 39-55, 2002.

[4] K. S. Kim and Q. Zou, "Model-less inversion-based iterative control for output tracking: Piezoelectric actuator example," in acc, (Seattle, Washington, USA), 2008.

[5] A. A. Tseng, A. Notargiacomo, and T. P. Chen, "Nanofabrication by scanning probe microscope lithography: A review," Journal of Vacuum Science \& Technology B: Microelectronics and Nanometer, vol. 23, pp. 877-894, May, 2005.

[6] A. Chimmalgi, C. P. Grigoropoulos, and K. Komvopoulos, "Surface nanostructuring by nano-/femtosecond laser-assisted scanning force microscopy," Applied Physics, vol. 97, p. 104319, 2005.

[7] $\ddot{A}$. Hoffmann, T. Jungk, and E. Soergel, "Cross-talk correction in atomic force microscopy," Review of Scientific Instrument, vol. 78, p. 016101, 2007.

[8] L. Y. Pao, J. A. Butterworth, and D. Y. Abramovitch, "Combined feedforward/feedback control of atomic force microscopes," in acc, (New York City, USA), 2007. 